\title{
HYDROXYCINNAMIC ACID DERIVATIVES AND FLAVONOL PROFILES OF MAQUI (Aristotelia chilensis) FRUITS
}

\author{
ANTONIETA RUIZ ${ }^{1,3}$, EDGAR PASTENE ${ }^{2}$, CAROLA VERGARA ${ }^{1}$, DIETRICH VON BAER ${ }^{1}$ \\ MARCIA AVELLO', CLAUDIA MARDONES ${ }^{I^{*}}$
}

\author{
${ }^{1}$ Instrumental Analysis Department, Faculty of Pharmacy, University of Concepción, P.O. Box 160-C, Concepción, Chile. \\ ${ }^{2}$ Pharmacy Department, Faculty of Pharmacy, University of Concepción, P.O. Box 160-C, Concepción, Chile. \\ ${ }^{3}$ Departamento de Ciencias Químicas y Recursos Naturales, Universidad de La Frontera, Casilla 54-D, Temuco, Chile
}

\begin{abstract}
Maqui (Aristotelia chilensis) is an edible berry native to Southern Chile. The anthocyanin profiles and concentrations of this fruit have been widely studied. The high concentration of anthocyanins confers a deep colour to the skin and pulp of maqui berries, and the biological activity of this fruit has been attributed to these compounds. However, other compounds such as flavonols and hydroxycinnamic acid derivatives in this fruit have not been studied to date.

The objective was to determine the hydroxycinnamic acid derivatives and flavonol profiles in maqui berries in order expand the knowledge base for this fruit.

Two extraction methodologies (solid phase extraction using a mixed mode cation exchange cartridge and selective precipitation), followed by HPLC-DADESI-MS/MS were applied to the extraction and identification of the main hydroxycinnamic acids and flavonols in the berries. Quantification was accomplished by using the first extraction procedure.

Low levels of hydroxycinnamic acid derivatives were detected in maqui berries, where 5-galloylquinic acid was the only quantifiable derivative. However, diverse and high levels of flavonols were detected in the fruit, the most relevant of which are myricetin-galactoside, quercetin-rutinoside, and quercetin-3galactoside. Nineteen other flavonols were identified, with total concentrations between 1.05 and $1.18 \mu \mathrm{mol} / \mathrm{g}$ fresh weight.

The diversity of flavonols makes the profiles and concentrations of these compounds significant, whereas hydroxycinnamic acids in maqui are not significant. The comparatively high levels of flavonols combined with the high concentration of anthocyanins contribute to the consideration of this native fruit from Chile as a "superfruit".
\end{abstract}

\section{INTRODUCTION}

Aristotelia chilensis (Molina) Stuntz (Elaeocarpaceae), also known as Chilean blackberry ${ }^{1}$, is a small tree that grows between Coquimbo and Aysén regions of Chile up to 2500 metres above sea level ${ }^{2}$. The fruit of this tree is an edible berry with a diameter of $5 \mathrm{~mm}$, which contains 2 seeds, where the pulp is intensely coloured. Maqui fruits have been traditionally used as a colorant as well as in natural medicine ${ }^{3}$ and maqui has thus been the topic of numerous studies ${ }^{1,4-12}$. Despite the extensive research, the profiles of flavonols and hydroxycinnamic acid derivatives $\left(\mathrm{HCAD}_{\mathrm{S}}\right)$ and their concentrations in maqui berries has not been documented. Prior research has focused primarily on the high anthocyanin content of this fruit ${ }^{4,8}$. The anthocyanin profile of maqui berries has been described, indicating the presence of 3 -glucoside, 3,5-diglucoside, 3-sambubioside, and 3-sambubioside-5-glucoside derivatives of delphinidin and cyanidin ${ }^{4,8}$, with a total anthocyanin concentration of 137.6 $\pm 0.4 \mathrm{mg} / 100 \mathrm{~g}$-fresh weight ${ }^{4}$. The use of maqui berries in functional food preparations, especially juices, based on their rich anthocyanin content and antioxidant activity has also been recently studied ${ }^{10,11,13,14}$. Only quercetin and myricetin were detected during the evaluation of the flavonol profile of maqui berry ${ }^{6}$; however, no derivatives of these compounds or conjugated forms have been described. Evaluation of the hydroxybenzoic acid content by the same authors ${ }^{6}$ revealed the presence of gentisic, ferulic, gallic, $p$-coumaric, synapic, and 4-hydroxybenzoic acids; however, as mentioned, there is no available information regarding the profiles and concentrations of HCADs in maqui fruit. However, the phenolic compounds in maqui leaves have been studied, where the presence of flavonoids, indolic alkaloids, coumarins, and triterpenoids has been reported ${ }^{5}$.

The antioxidant capacity of maqui fruit was compared with that of other Chilean berries such as calafate (Berberis microphylla) and murtilla (Ugni molinae) using oxygen radical absorbance capacity methodology (ORAC). The reported antioxidant levels in $\mu$ mol Trolox equivalents/100 g (fresh weight) were 25662, 19850, 10770, and 8869 for calafate, maqui, murtilla, and blueberries, respectively; these levels are all higher than those detected for other widely consumed fruits such as apples, pears, apricots, peaches, and plums ${ }^{15}$.

The biological effects of maqui fruit extracts has been documented based on in vitro studies, with claims of anti-inflammatory effects ${ }^{7,16}$, antioxidant properties $^{6,7,15}$, antiatherogenic activity ${ }^{17}$, cardioprotective ${ }^{1}$ and hypoglycemic effects $^{9}$, as well as antihaemolytic properties ${ }^{18}$. Based on these studies, maqui fruit is considered as a potential source of bioactive compounds; however, to fully understand and exploit the potential of maqui as functional fruit, the content and profiles of flavonols and $\mathrm{HCAD}_{\mathrm{S}}$ must be elucidated.

As an analytical challenge, the identification and quantification of other minor phenolic compounds (i.e., flavonols or $\mathrm{HCAD}_{\mathrm{S}}$ ) may be hampered by the extremely high concentrations of anthocyanins in maqui. The absorption spectra of the natural pigments are characterized by an important band in the UV region that interferes with the detection of these phenolic compounds ${ }^{19}$. More selective extraction methodologies are required in order to obtain anthocyanin-free fractions and improve the identification and quantification of non-anthocyanic phenolic compounds in maqui berries.

Based on the aforementioned considerations, the overarching aim of the present study is to expand the knowledge base about maqui berries and their potential as functional fruit. To accomplish this task, we evaluated the profiles and contents of HCADs and flavonols in samples of this fruit. Improved detection of these phenolic compounds was achieved by two complementary extraction procedures, one based on solid phase extraction SPE and the other based on selective precipitation, both followed by HPLC-DAD-ESI-MS/MS analysis.

\section{EXPERIMENTAL}

\section{Reagents and standards}

Commercial standards of 3-caffeoylquinic (98.87\%), 4-caffeoylquinic, and 5-caffeoylquinic acids $(98.28 \%)$ were obtained from Phytolab (Vestenbergsgreuth, Germany) and standards of myricetin $(>85 \%)$, quercetin $(>98 \%)$, kaempferol $(>90 \%)$, quercetin-3-rutinoside ( $>95 \%)$, quercetin3-galactoside $(>97 \%)$, quercetin-3-glucoside $(>90 \%)$, and quercetin-3rhamnoside ( $>85 \%$ ) were obtained from Sigma-Aldrich (Steinheim, Germany). Formic acid, hydrochloric acid, and ammonia (25\%) were obtained from Merck (Darmstadt, Germany). All other solvents (water, acetonitrile, and methanol) were HPLC grade and were purchased from Merck. Mixed phase MCX cartridges were obtained from Waters (Milford, USA).

Sample material

Maqui fruits (Aristotelia chilensis) were collected on the campus of the University of Concepción (UDEC) in Concepción (Bío-Bio region), Los Robles (Manzanares, Araucanía region), and Lican Ray (Araucanía region) in Chile during summer 2010 and 2011.

Extraction of hydroxycinnamic acid derivatives and flavonols

Two complementary extraction procedures were carried out in order to obtain a selective extraction for both groups of compounds (flavonols and HCADs). The first procedure was carried out based on a previously described method $^{19}$ comprising liquid-solid extraction using methanol acidified with 
formic acid, followed by a clean-up step using Oasis MCX cartridges. Oasis MCX is a mixed-mode cation exchange reversed phase, which allows elimination of sugars and anthocyanins ${ }^{19,20}$.

The second extraction method comprised a fractional precipitation procedure in which $5 \mathrm{~g}$ of the dry extract obtained using acidified methanol (with $0.5 \% \mathrm{HCl}$ ) was dissolved in $250 \mathrm{~mL}$ of water with $5 \%$ formic acid. Using a peristaltic pump, this solution was loaded on Sepabeads SP-850 absorber resin (mean pore size: $38 \AA$; Supelco, Bellefonte, PA) that was packed in a glass column $(40 \mathrm{~mm}$ i.d. $\times 300 \mathrm{~mm})$ pre-conditioned with acidic water. A washing step was performed using $3 \mathrm{~L}$ of water. Phenolic compounds were eluted with 1 $\mathrm{L}$ of $5 \%$ formic acid in methanol and the obtained extract was pre-concentrated and lyophilized. One gram of this extract was re-dissolved in $50 \mathrm{~mL}$ of $0.08 \%$ trifluoracetic acid (TFA) in ethanol and centrifuged to eliminate the insoluble material. Subsequently, $50 \mathrm{~mL}$ of $n$-pentane was added and the precipitate was collected by centrifugation. Precipitation with pentane was repeated two times. Finally, the precipitate was redissolved in $0.08 \%$ TFA in a water/acetonitrile $70 / 30$ mixture and loaded on a MCI gel $(2.5 \times 15 \mathrm{~cm})$ column. Elution was carried out with $0.08 \%$ TFA in water/acetonitrile $30 / 70(\mathrm{v} / \mathrm{v})$ at a flow rate of $12 \mathrm{~mL} / \mathrm{min}$. Under these conditions, anthocyanins were eluted at $10 \mathrm{~min}$, while flavonols and hydroxycinnamic acids were recovered after $45 \mathrm{~min}$.

Evaluation of the flavonol and HCAD profiles of maqui was accomplished by applying both extraction methods in parallel, followed by HPLC-DAD-ESIMS/MS analysis.

\section{HPLC-DAD-ESI-MS/MS chromatographic conditions}

Chromatographic analyses of flavonols and HCADs in maqui fruit extracts was carried out with a Shimadzu HPLC NEXERA system (Kyoto, Japan) equipped with a quaternary LC-30AD pump, a DGU-20A $\mathrm{A}_{5 \mathrm{R}}$ degasser unit, a CTO-20AC column oven, a SIL-30AC autosampler, a CBM-20A controller system, and a UV-vis diode array (DAD) SPD-M20A detector, coupled in tandem with a Q-Trap LC/MS/MS 3200 Applied Biosystems MDS Sciex (California, USA) detector. Instrument control and data collection was performed by using a CLASS-VP DAD Shimadzu Chromatography Data
System and Analyst Software (Version 1.5.2).

The procedure described by Ruiz ${ }^{19}$ was used as the chromatographic technique for identification and quantification of flavonols and HCADs. A C18 column (Kromasil $250 \times 4.6 \mathrm{~mm}, 5 \mu \mathrm{m}$ ) and a C18 pre-column (Nova-Pak Waters, $22 \times 3.9 \mathrm{~mm}, 4 \mu \mathrm{m}$ ) (Milford, MA) were used for separation at $30^{\circ} \mathrm{C}$. Gradient elution was performed by varying the mobile phase from $15 \%$ to $25 \%$ acetonitrile in $14 \mathrm{~min}$, from $25 \%$ to $35 \%$ for $11 \mathrm{~min}$, from $35 \%$ to $100 \%$ for $1 \mathrm{~min}$, and from $100 \%$ to $15 \%$ for $1 \mathrm{~min}$ with a final stabilization period of $10 \mathrm{~min} . \mathrm{MS} / \mathrm{MS}$ was performed in negative ionization mode using a collision energy of $-5 \mathrm{~V}$, ionization voltage of $-4000 \mathrm{~V}$, capillary temperature of $450^{\circ} \mathrm{C}$, and the $\mathrm{N}_{2}$ nebulizer was set to $15 \mathrm{psi}$. Identification of the analytes was carried out by comparison of their retention time $\left(t_{\mathrm{R}}\right)$ and spectral (MS/MS and UV) characteristics with those of the respective commercially available standards. Quantification was performed by using a DAD chromatogram extracted at 320 $\mathrm{nm}$ for flavonols and at $360 \mathrm{~nm}$ for HCADs. External calibration curves were constructed using 3-caffeoylquinic acid for HCAD determination and using myricetin, quercetin, and kaempferol for quantitative flavonol determination. All results are expressed as $\mu \mathrm{mol} / \mathrm{g}$ fresh weight.

\section{RESULTS AND DISCUSSION}

\section{HCADs}

Only two HCADs were identified in the maqui extracts, based on the UV and MS/MS spectra. These compounds were only detected when the SPE extraction procedure was applied, whereas no HCADs were detected in the extract purified by precipitation. Figure 1A shows the HPLC-DAD chromatogram of the maqui extract at $320 \mathrm{~nm}$ obtained by the SPE methodology. The compound with the most abundant signal (peak 1) shows a UV maximum at $331 \mathrm{~nm}$, but this signal was not detected under the MS/MS conditions used for HCADs. A similar situation was observed for the compounds corresponding to signals 3,5 , and 7 , which also presented UV absorption at $320 \mathrm{~nm}$; however, these species could not be assigned as HCADs based on the UV and MS/MS spectra.

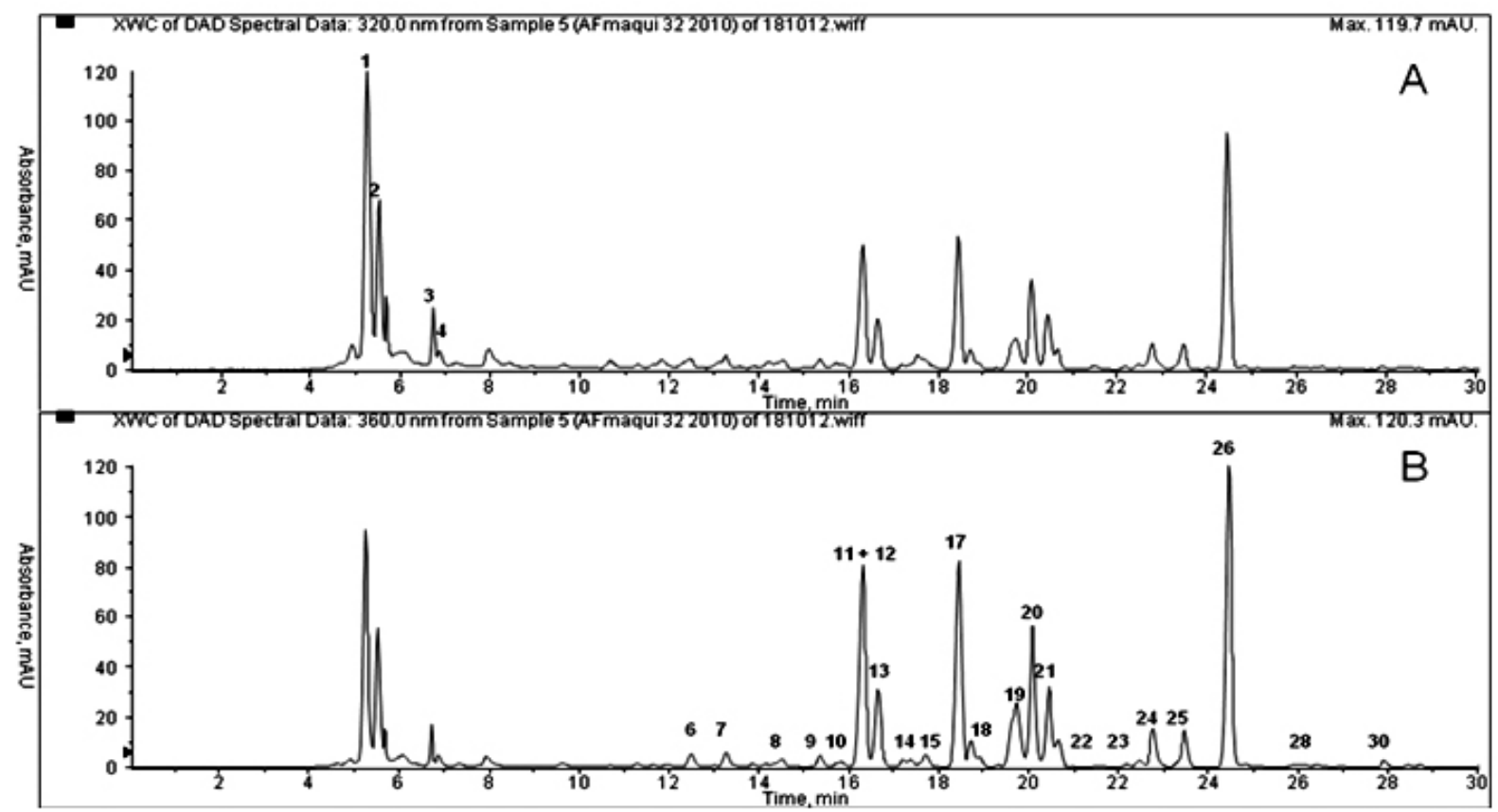

Figure 1: HPLC-DAD chromatogram of flavonols and HCAD in maqui fruits obtained using SPE with mixed phase cartridges. (A) $320 \mathrm{~nm}$, (B) $360 \mathrm{~nm}$. Peaks identities are listed in Table 1. 


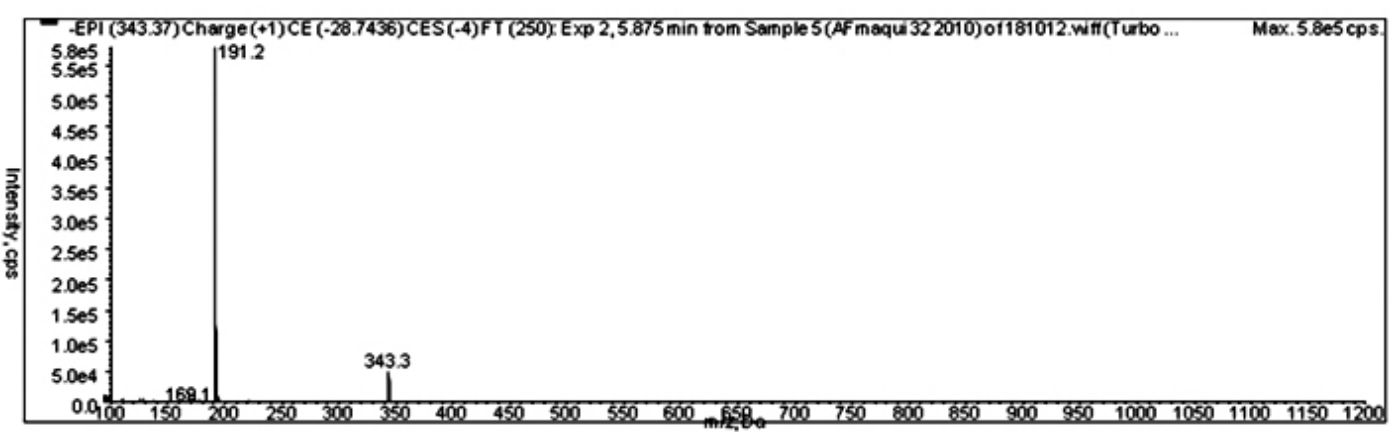

Figure 2: MS/MS spectra of galloylquinic acid in maqui fruits.

Detection of these species was also attempted by using positive ionization in the MS/MS analysis; however MS/MS signals were not detected, making it impossible to identify these compounds. On the other hand, a molecular ion $[\mathrm{M}-\mathrm{H}]^{-}$at 343 and two product ions at $\mathrm{m} / \mathrm{z} 191$ and 169 (Figure 2) were observed for the most relevant HCAD signal (peak 2) with a maximum UV absorption at $333 \mathrm{~nm}$. On the basis of these characteristics, this compound was tentatively assigned as 5-galloylquinic acid, the spectrum of which is congruent with the data presented by Clifford ${ }^{21}$. Peak 4 was assigned as protocatechuic-4glucosidic acid based on the MS/MS data in which a pseudomolecular ion peak [M-H] was observed at $m / z 315$, which is congruent with the data presented by Rodriguez-Medina ${ }^{22}$. The retention times and spectroscopic characteristics of these compounds are detailed in Table 1.

Table 1: Flavonols and hydroxycinnamic acid derivatives in maqui fruits

\begin{tabular}{|c|c|c|c|c|c|c|}
\hline Peak & Compound & $\mathbf{t}_{\mathrm{R}}(\min )$ & $\lambda \max (\mathbf{n m})$ & {$[\mathbf{M}-\mathbf{H}]^{-}$} & Product ions & $\begin{array}{c}\text { Kind of } \\
\text { Extraction }\end{array}$ \\
\hline 1 & n.i. & 5,2 & 331 & - & - & a \\
\hline 2 & 5-galloylquinic acid & 5,5 & 333 & 343,3 & 191,$2 ; 169,1$ & $\mathrm{a}$ \\
\hline 3 & n.i. & 6,7 & 317 & - & - & $\mathrm{a}$ \\
\hline 4 & protocatechuic-4-glucosidic acid & 7,3 & no & 315 & - & $\mathrm{a}$ \\
\hline 5 & n.i. & 12,3 & 276 & - & - & $\mathrm{b}$ \\
\hline 6 & quercetin-hexoside & 12,7 & 359 & 463,3 & 300,2 & $\mathrm{a}$ \\
\hline 7 & n.i. & 13,2 & 278 & - & - & $\mathrm{a}, \mathrm{b}$ \\
\hline 8 & myricetin-galloylhexoside & 14,7 & no & 631,4 & 479,$3 ; 316,3$ & $\mathrm{a}, \mathrm{b}$ \\
\hline 9 & myricetin-rutinoside & 15,5 & no & 625,6 & 316,4 & $\mathrm{a}$ \\
\hline 10 & quercetin-hexoside & 16,0 & 354 & 463,3 & 300,3 & a \\
\hline 11 & myricetin-galactoside & 16,3 & 359 & 479,3 & 316,2 & $\mathrm{a}, \mathrm{b}$ \\
\hline 12 & myricetin-glucoside & 16,6 & 355 & 479,3 & 316,2 & $\mathrm{a}, \mathrm{b}$ \\
\hline 13 & quercetin-3-rhamnoside & 17,2 & no & 447,2 & 300,1 & $\mathrm{a}$ \\
\hline 14 & quercetin-pentoside & 17,5 & no & 433,3 & 300,1 & $\mathrm{a}$ \\
\hline 15 & quercetin-galloylgalactoside & 17,8 & 359 & 615 & 463,$3 ; 300,2$ & $\mathrm{a}, \mathrm{b}$ \\
\hline 16 & quercetin-galloylglucoside & 18,4 & no & 615,4 & 463,$3 ; 300,2$ & $\mathrm{~b}$ \\
\hline 17 & quercetin-3-rutinoside & 18,4 & 355 & 609,4 & 300,2 & $\mathrm{a}$ \\
\hline 18 & myricetin-pentoside & 19,1 & 347 & 449,3 & 316,2 & $\mathrm{a}, \mathrm{b}$ \\
\hline 19 & quercetin & 19,9 & 367 & 301 & - & $\mathrm{a}, \mathrm{b}$ \\
\hline 20 & quercetin-3-galactoside & 20,1 & 355 & 463,3 & 300,2 & $\mathrm{a}, \mathrm{b}$ \\
\hline 21 & quercetin-3-glucoside & 20,5 & 354 & 463,3 & 300,2 & $\mathrm{a}$ \\
\hline 22 & kaempferol-hexoside & 20,8 & no & 447,2 & 285,3 & $\mathrm{a}$ \\
\hline 23 & myricetin +283 & 22,5 & no & 599,3 & 316,2 & a \\
\hline 24 & quercetin-pentoside & 22,9 & 355 & 433,3 & 300,2 & $\mathrm{a}, \mathrm{b}$ \\
\hline 25 & quercetin-pentoside & 23,6 & 352 & 433,3 & 300,2 & $\mathrm{a}, \mathrm{b}$ \\
\hline 26 & n. i. & 24,5 & 346 & 329,3 & 314,$2 ; 271,2$ & $\mathrm{a}$ \\
\hline 27 & myricetin-6"'-O-hexosil-C-hexoside & 24,9 & no & 599,3 & 479,$4 ; 316,2$ & $\mathrm{~b}$ \\
\hline 28 & quercetin- $6^{\prime \prime}$ - $O$-hexosil-C-hexoside & 26,2 & no & 583,3 & 463,$3 ; 300,2$ & $\mathrm{a}, \mathrm{b}$ \\
\hline 29 & n.i. & 27,9 & 376 & - & - & $\mathrm{b}$ \\
\hline 30 & quercetin & 28,1 & no & 301,3 & - & $\mathrm{a}, \mathrm{b}$ \\
\hline
\end{tabular}

(a) SPE with MCX cartridges, (b) extraction by precipitation. n.i.: not identified. 
Flavonols

A diversity of flavonols was detected in the maqui fruit extracts, where 22 flavonols were identified, corresponding to 14 quercetin derivatives, 7 myricetin derivatives, and 1 kaempferol derivative (Table 1). Both extraction methods (SPE and selective precipitation) functioned complementarily for detection of these compounds. Figure 1B shows the flavonol profile of maqui obtained by the SPE procedure, while Figure 3B shows the flavonol profile obtained by selective precipitation.

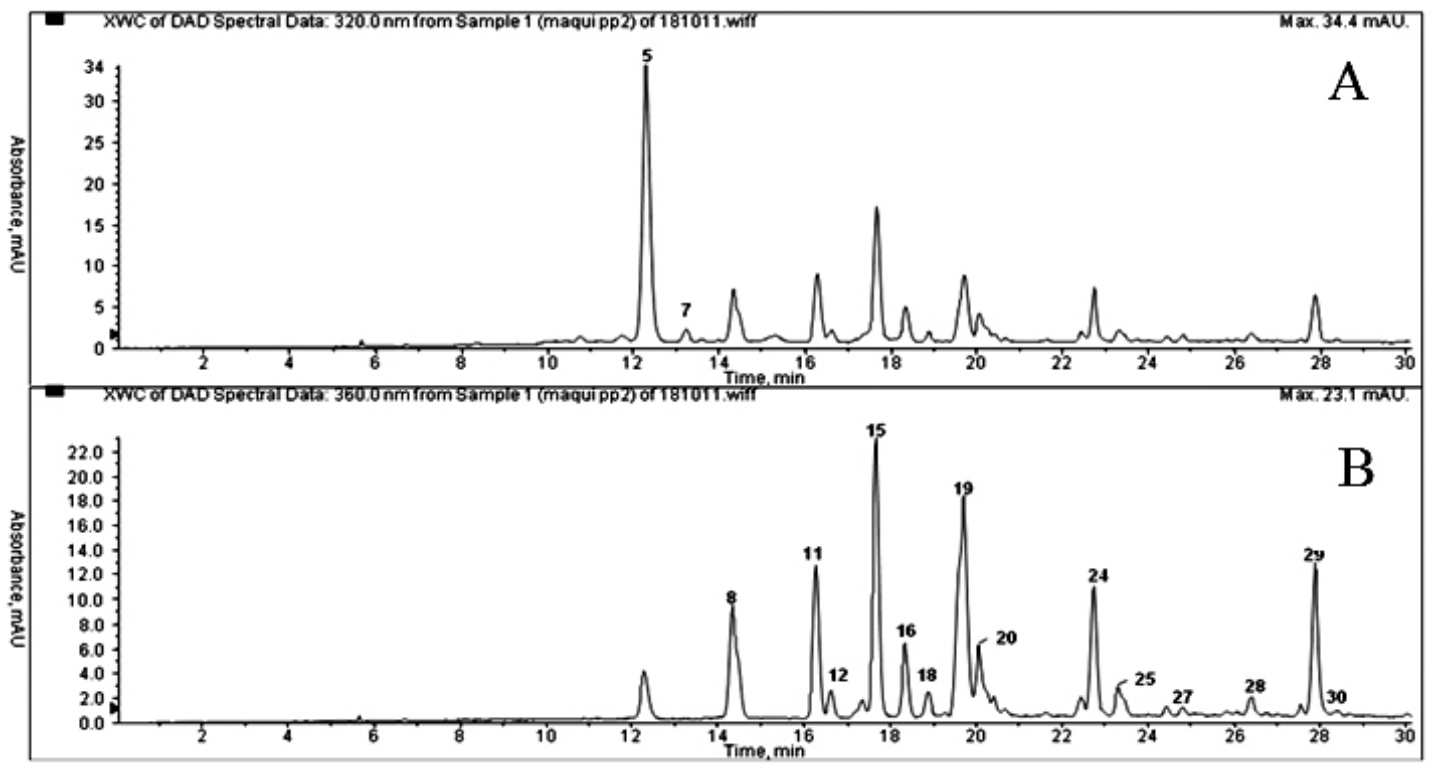

Figure 3: HPLC-DAD chromatogram of flavonols and HCAD in maqui fruits obtained using precipitation extraction. (A) 320 $\mathrm{nm}$, (B) $360 \mathrm{~nm}$. Peaks identities are listed in Table 1.

The quercetin derivatives detected in this fruit included four hexosides, one rhamnoside, one rutinoside, four pentosides, two galloylhexoside derivatives, one unidentified derivative, and a free aglycone. The identities were assigned based on the respective mass spectrometric fragmentation patterns and the UV spectra. A pseudomolecular ion was observed at $\mathrm{m} / \mathrm{z} 615$ for both galloylhexoside derivatives, with product ions at $\mathrm{m} / \mathrm{z} 463$ and 300 . In this case, the compound identities were assigned by also considering the fragmentation pattern described by Scoparo $^{23}$, as well as on the basis of the elution order. The first compound eluted (peak 15) was tentatively assigned as quercetin-galloylgalactoside, while the second peak eluted was assigned as quercetin-galloylglucoside (peak 16); both compounds have previously been detected in blueberries ${ }^{24}$. The myricetin derivatives included one rutinoside, one hexoside, one pentoside, one galloylhexoside, and one unidentified derivative. A pseudomolecular ion [M-H] at $\mathrm{m} / \mathrm{z} 631$ and product ions at $\mathrm{m} / \mathrm{z}$ 479 and 316 were detected for the galloylhexoside derivative, as well as for the other galloylated compounds. The compound was tentatively identified according to the fragmentation pattern described by Scoparo ${ }^{23}$.

Myricetin and quercetin derivatives were detected, characterized by respective molecular ions at $\mathrm{m} / \mathrm{z} 599.3$ and 583.3 and product ions at $\mathrm{m} / \mathrm{z} 479$ and 316 for the myricetin derivative and at 463 and 300 for the quercetin derivative. These fragmentation patterns have been previously described for $6^{\prime \prime}-O$-hexosil-C-hexosil derivatives of flavones ${ }^{25}$, but not for flavonols. Considering that both $[\mathrm{M}-\mathrm{H}]^{-}$ions correspond to myricetin and quercetin derivatives and that the lost fragment in both cases are the same as described for the flavones, we propose that the compounds detected in the maqui extract may correspond to myricetin- 6 "'-O-hexosil-C-hexosil (peak 27) and quercetin6"-O-hexosil-C-hexosil (peak 28).

A myricetin derivative (peak 23) with a pseudomolecular ion $[\mathrm{M}-\mathrm{H}]^{-}$at $\mathrm{m} / \mathrm{z} 599$ and a product ion at $\mathrm{m} / \mathrm{z} 383$ was detected; however, the identity of the conjugate was not unambiguously defined, and the compound was generally assigned as a myricetin-derivative. Only one kaempferol derivative was detected, corresponding to kaempferol-hexoside (peak 22).

The SPE methodology using MCX cartridges facilitated detection of 20 flavonols, one of which was not identified (peak 26 in Figure 1B). The most abundant compounds obtained using this extraction procedure at $359 \mathrm{~nm}$ corresponded to myricetin-galactoside (peak 11), quercetin-rutinoside (peak 17 ), and quercetin-3-galactoside (peak 20).
However, the selective precipitation methodology facilitated detection of only 16 flavonols, coinciding with 13 flavonol signals already identified in the first extraction, whereas the other 3 were not detected in the SPE procedure (peaks 5, 7, and 29). In this case, the most abundant compounds corresponded to quercetin-galloylhexoside (peak 15), quercetin (peak 19), and quercetinpentoside (peak 24).

These two extraction methodologies evidently gave rise to different flavonol and HCAD profiles for maqui fruits. This approach is an analytical complementary strategy, which allows the extraction of flavonols and HCADs to facilitate identification, and can be used as a tool for isolation of these types of compounds from other fruits rich in anthocyanins.

\section{Concentrations of HCAD and flavonols in maqui fruits}

Quantification of the flavonols and HCADs in the maqui fruit extracts was performed with the extract obtained by SPE. The extract obtained via the precipitation methodology was not used for quantification because the extract was prepared only for semi-preparative purposes for identification. Moreover, the complex steps required in this procedure would increase the uncertainty of any quantitative methodology.

The only quantifiable HCAD in maqui fruit extract was 5-galloylquinic acid, with levels between 0.05 and $0.11 \mathrm{mmol} / \mathrm{g}$; this was considered as the total concentration of HCADs in this fruit. This level is lower than those reported for other endemic berries from Chilean Patagonia, such as calafate ${ }^{19}$ and other Chilean berries such as Ribes magellanicum $(3.53 \mathrm{mmol} / \mathrm{g})$ and Ribes cucullatum $(1.41 \mathrm{mmol} / \mathrm{g})^{26}$, as well as other berry crops like black and red currant ${ }^{27}$. However, the HCAD concentration is of the same order of magnitude as reported for other berries such as Ribes nigrum ${ }^{27}$. The level of HCADs in maqui fruit is also comparable to that reported for Ribes rubrum (0.006-0.3 $\mu \mathrm{mol} / \mathrm{g})^{27}$ and lower than the levels reported for, Vaccinium spp (0.97-3.21 $\mathrm{mmol} / \mathrm{g})^{28}$, and Vaccinium corimbosum L. $(0.73-1.91 \mathrm{mmol} / \mathrm{g})^{27}$.

The flavonols detected in maqui extract corresponded to myricetin and quercetin derivatives, of which the most important were quercetin-3-rutinoside $(0.22-0.42 \mu \mathrm{mol} / \mathrm{g})$ and quercetin-3-galactoside $(0.17-0.25 \mu \mathrm{mol} / \mathrm{g})$, with a total flavonol concentration of between 1.05 and $1.14 \mu \mathrm{mol} / \mathrm{g}$. The total concentrations of $\mathrm{HCAD}_{\mathrm{S}}$ and flavonols are summarized in Table 2. The flavonol content of maqui fruit is higher than the HCAD content, which is opposite to the trend reported for calafate fruits in which $\mathrm{HCAD}_{\mathrm{S}}$ are predominant when anthocyanins, which represent the most abundant group of phenolic compounds in both fruits, are not considered ${ }^{8}$. 
Table 2: Total concentrations of flavonols and hydroxycinnamic acid derivatives (HCADs) in maqui fruits.

\begin{tabular}{|c|c|c|}
\hline Sample & $\begin{array}{c}\text { Total } \\
\text { HCADs } \\
(\boldsymbol{\mu m o l} / \mathbf{g})\end{array}$ & $\begin{array}{c}\text { Total flavonols } \\
(\boldsymbol{\mu m o l} / \mathbf{g})\end{array}$ \\
\hline Maqui Concepción (Bio-bio region) & 0.07 & 1.14 \\
\hline Maqui Los Robles (Araucania region) & 0.05 & 1.18 \\
\hline Maqui Lican Ray (Araucanía region) & 0.11 & 1.05 \\
\hline
\end{tabular}

The total concentration of flavonols in maqui berries $(1.05-1.18 \mu \mathrm{mol} / \mathrm{g})$ is higher than the levels reported for other berry species, such as blackberry $(0.86$ $\mu \mathrm{mol} / \mathrm{g})$, rowanberry $(0.77 \mu \mathrm{mol} / \mathrm{g})$, blackcurrant $(0.67 \mu \mathrm{mol} / \mathrm{g})$, strawberry $(0.03 \mu \mathrm{mol} / \mathrm{g})^{29}$, and Vaccinium corymbosum $(0.05-0.11 \mathrm{mmol} / \mathrm{g})^{27}$. This finding is very interesting in terms of the nutraceutical potential of this fruit, especially when the very high anthocyanin concentration of maqui fruit is considered $\mathrm{d}^{4,30}$ in conjunction with the interesting levels of flavonols found in the present study.

\section{CONCLUSIONS}

The analytical strategy presented herein, in which selective precipitation and a SPE method were complementarily combined, followed by HPLC-DADESI-MS/MS, facilitated the selective extraction and identification of HCADs and flavonols from maqui fruits. The SPE method facilitated extraction of a larger number of compounds than the precipitation method, and it was also more suitable for quantitative purposes.

Galloylquinic acid was the most abundant HCAD detected in maqui fruits, whereas protocatechuic-4-glucosidic acid was also detected. The other observed signals were not fragmented under the optimal conditions used for HCAD. For this reason, these compounds were not assigned as HCADs. Notably, gallic acid was also detected as a conjugate with flavonols such as quercetin and myricetin.

The bioactive compounds in maqui berries, especially the higher content of flavonols in this fruit relative to the levels reported for other berries, contribute to the consideration of this native fruit from Chile as a "superfruit". The data presented herein represent an expansion of the knowledge-base about phenolic compounds in maqui fruit where the profiles of two new groups (flavonoids and HCADs) of compounds are documented, in addition to that of anthocyanins.

\section{ACKNOWLEDGMENTS}

Authors would like to thank FONDECYT Chile, Grant 1140439 for financial support.

\section{REFERENCES}

1. C. Cespedes, M. El-Hafidi, N. Pavon, J. Alarcón, Food Chem. 107, 820, (2008)

2. Fundación Flora Chilena Darian Stark, Enciclopedia de la Flora Chilena. URL [http://www.florachilena.cl/especies.php?id=352]; accessed December 2014.

3. Hoffman Flora Silvestre de Chile zona araucana, árboles, arbustos y enredaderas leñosas ( $5^{\text {th }}$ Edn.). Ediciones Fundación Claudio Gay, Santiago, 2005.

4. M .Escribano-Bailón, C. Alcalde-Eon, O. Muñoz, J. Rivas- Gonzalo, C. Santos-Buelga, Phytochem. Anal 17, 8, (2006)

5. M. Suwalsky, P. Vargas, M. Avello, F. Villena, C. Sotomayor, Int. J. Pharm. 363, 85, (2008)

6. Céspedes, M. Valdez-Morales, J. Avila, M. El-Hafidi, J. Alarcón, O. Paredes-Lopez, Food Chem. 119, 886, (2010)

7. Céspedes, J. Alarcón, J. Avila, A. Nieto, BLACPMA 9, 127, (2010)

8. Ruiz, I. Hermosin-Gutierrez, C. Mardones, C. Vergara, E. Herlitz, M. Vega, C. Dorau, P. Winterhalter, D. von Baer, J. Agric. Food Chem. 58, 6081, (2010)

9. L. Rojo, D. Robnicky, S. Logendra, A. Poulev, P. Rojas-Silva, P. Kuhn, R. Dorn, M. Grace, M. Lila, I. Raskin, Food Chem. 131, 387, (2012)

10. Girones-Vilaplana, P. Valentao, D. Moreno, F. Ferreres, C. Garcia-Viguera, P. Andrade, J. Agric. Food Chem. 60, 6571, (2012)

11. Girones-Vilaplana, P. Mena, C. Garcia-Viguera, LWT-Food Sci. Technol. 47, 279, (2012)

12. J. Tanaka, T. Kadekaru, K. Ogawa, S. Hitoe, H. Shimoda, H. Hara, Food
Chem. 139, 129, (2013)

13. Girones-Vilaplana, A. Calin-Sanchez, D. Moreno, A. CarbonellBarrachina, C. Garcia-Viguera, Food Chem. 173, 1228, (2015)

14. Girones-Vilaplana, D. Villano, D. Moreno, C. Garcia-Viguera, Int. J. Food Sci. Nutr. 64, 897, (2013)

15. Speisky, C. López-Alarcón, M. Gómez, J. Fuentes, C. Sandoval-Acuña, J. Agric. Food Chem. 60, 8851, (2012)

16. O. Muñoz, P. Christen, S. Cretton, N. Backhouse, V. Torres, O. Correa, E. Costa, H. Miranda, O. Delporte, J. Pharm. Pharmacol. 63, 849, (2011)

17. S. Miranda-Rottman, A. Aspillaga, D. Pearez, L. Vasquez, A. Martinez, F. Leighton, J. Agric. Food Chem. 50, 7542, (2002)

18. M. Rubilar, C. Jara, Y. Poo, F. Acevedo, C. Gutierrez, J. Sineiro, C. Shene, J. Agric. Food Chem. 59, 1630, (2011)

19. Ruiz, C. Mardones, C. Vergara, I. Hermosín-Gutiérrez, D. von Baer, P. Hinrichsen, R. Rodriguez, D. Arribillaga, E. Dominguez, J. Chrom. A 1281, 38, (2013)

20. N. Castillo-Munoz, S. Gomez-Alonso , E. Garcia-Romero, I. HermosinGutierrez, J. Agric. Food Chem. 55, 992, (2007)

21. Clifford, S. Stoupi, N. Kuhnert, J Agric. Food Chem. 55, 2797, (2007)

22. Rodriguez-Medina, A. Segura-Carretero, A. Fernandez-Gutierrez, J. Chrom. A 1216, 4736, (2009)

23. Scoparo, L. de Souza, N. Dartora, G. Sassaki, P. Horin, M. Iacomini, J. Chrom. A 1222, 29, (2012)

24. Cho, L. Howard, R. Prior, J. Clark, J. Sci. Food Agric. 85, 2149 (2005)

25. F. Ferreres, A. Gil-Izquierdo, P. Andrade, P. Valentao, F. Tomás Barberán, J. Chrom. A 1161, 214, (2007)

26. A. Ruiz, L. Bustamante, C. Vergara, D. von Baer, I. Hermosín-Gutiérrez, L. Obando, C. Mardones, Food Chem. 167, 84 (2015)

27. V. Gavrilova, M. Kajd-Zanoska, V. Gjamovski, M. Stefova, J. Agric. Food Chem. 59, 4009, (2011)

28. Rodriguez-Mateos, T. Cifuentes-Gomez, S. Tabatabaee, C. Lecras, J. Spencer, J. Agric. Food Chem. 23, 5772, (2012)

29. M. Mikulic-Petkovsek, A. Slatnar, F. Stampar, R. Veberic, Food Chem. 135, 2138, (2012)

30. Fredes, G. Yousef, P. Robert, M. Grac, M. Lila, M. Gomez, M. Gebauer, G. Montenegro, J. Sci. Food Agric. 94, 2639, (2014) 